\title{
Inhibición del acero galvanizado en medio salino mediante tricloruro de cerio
}

\author{
F. Serrano ${ }^{(*)}$ y J.J. de Damborenea ${ }^{(*)}$
}

\begin{abstract}
Resumen Se estudia la adición de pequeñas cantidades de tricloruro de cerio, como inhibidor de la corrosión de aceros galvanizados, en disoluciones aireadas de $\mathrm{NaCl}$ al 3,5\%. Los resultados encontrados hasta la fecha, obtenidos mediante técnicas electroquímicas y ensayos acelerados en laboratorio, muestran una clara reducción de la velocidad de disolución del cinc. Del análisis de los diagramas de polarización y de la superficie del electrodo, se deduce, de acuerdo con la bibliografía, que el cerio se comporta como un inhibidor catódico, bloqueando los centros de reducción de oxígeno disuelto. Los ensayos de inmersión alternada revelaron un excelente comportamiento de las probetas pretratadas en disoluciones que contenían cloruros de cerio, frente a aquellas que carecían de tratamiento. La formación de una película rica en cerio es la responsable del aumento de la capacidad protectora del acero galvanizado. Estos resultados confirman las posibilidades del cerio como posible sustituto de los tratamientos basados en cromatos, muy efectivos en estos materiales, pero altamente contaminantes.
\end{abstract}

Palabras clave: Cerio. Inhibidores. Acero galvanizado. Técnicas electroquímicas. Corrosión.

\section{Galvanised steel inhibition in saline medium by cerous chloride}

\begin{abstract}
The addition of small amounts of cerous chloride, as inhibitor of hot dip galvanised steel corrosion in aerated $3,5 \% \mathrm{NaCl}$ solutions is studied. The results found so far, carried out by electrochemical techniques and accelerated tests, show a clear decrease in the zinc dissolution rate. The analysis of polarization curves and specimen surface suggest, in accordance with literature, that cerium acts as a cathodic inhibitor, blocking the oxygen reduction centres. Alternate immersion tests developed an excellent behaviour of specimens pre-treated in cerium containing solutions, compared to those without treatments. A rich cerium film is responsible of the improvement in the protective properties. These results prove the possibilities of cerium as an alternative treatment to those based on chromates, which are very effective on these materials but highly toxic.
\end{abstract}

Keywords: Cerium. Inhibitors. Galvanised steel. Electrochemical techniques. Corrosion.

\section{INTRODUCCIÓN}

Los recubrimientos de cinc, en todas sus diferentes modalidades, sobre aleaciones férreas son ampliamente conocidos y utilizados en la industria. A pesar de comportarse como un metal más activo, la vida media de sus recubrimientos es bastante mayor que la del acero desnudo, debido a la formación de una película de óxido protectora sobre su superficie.

Sin embargo, a pesar de estas excelentes propiedades, es frecuente el tratamiento de las superficies de estos materiales con el fin de mejorar su resis-

(*) Centro Nacional de Investigaciones Metalúrgicas, CENIM (CSIC), Avda. de Gregorio del Amo, 8. 28040-Madrid (España). tencia a la corrosión. Uno de los tratamientos más utilizados es la formación de capas de conversión mediante el empleo de formulaciones basadas en compuestos de $\mathrm{Cr}$ (VI).

En estos casos, el aumento de la protección es notable y proporcional al espesor de la capa formada (normalmente de 0,5-3 $\mu \mathrm{m}$ ), además de constituir una buena base para el anclaje de pinturas (1). Sin embargo, los compuestos de cromo son substancias altamente tóxicas, siendo la especie $\mathrm{Cr}^{6+}$ un fuerte agente cancerígeno, además de ser una amenaza medioambiental cuando está presente en las aguas residuales.

La necesidad de encontrar alternativas más aceptables, desde el punto de vista ecológico, ha llevado a la investigación de sales de elementos lantánidos, en especial de cerio, como especies 
efectivas en la inhibición de la corrosión de varias aleaciones de aluminio (2-4) y aceros inoxidables (5-6). El cerio se comporta, en estos casos, como inhibidor catódico, bloqueando los centros de reducción de oxígeno debido a la precipitación de un óxido y/o hidróxido causado por el aumento local del $\mathrm{pH}$.

En este trabajo se presentan los primeros resultados obtenidos por los autores, sobre el comportamiento inhibidor del cerio en aceros galvanizados, añadiéndose de esta forma a los escasos estudios existentes en la bibliografía sobre esta materia (7).

\section{PROCEDIMIENTO EXPERIMENTAL}

\subsection{Materiales}

Como material de base, se han utilizado chapas de acero suave de $9 \times 5 \times 0,1 \mathrm{~cm}$, galvanizadas por inmersión en baño de zinc fundido a $450{ }^{\circ} \mathrm{C}$, y tiempo de inmersión de $1 \mathrm{~min}$.

Los ensayos de corrosión se realizaron por inmersión de las probetas en una disolución de $\mathrm{NaCl}$ al 3,5\% en masa y saturadas de oxígeno, preparadas a partir de reactivo químicamente puro.

Como inhibidor se ha empleado $\mathrm{CeCl}_{3} \cdot 7 \mathrm{H}_{2} \mathrm{O}$, ensayándose cuatro concentraciones diferentes $(100,200,500$ y $1.000 \mathrm{ppm})$.

Determinadas probetas fueron pretratadas mediante inmersión completa en disoluciones aireadas de $\mathrm{NaCl}$ al 3,5 \%, que contenían 1.000 ppm de inhibidor, durante $60 \mathrm{~h}$. La temperatura se mantuvo en $23 \pm 1{ }^{\circ} \mathrm{C}$.

\subsection{Ensayos de inmersión alternada}

Se ha desarrollado un ensayo acelerado de inmersión alternada en el que las probetas, colocadas sobre un bastidor, penetran y salen del medio agresivo durante cortos períodos de tiempo. Este ensayo, que representa la zona de salpicadura de agua de mar, ha permitido evaluar los pretratamientos descritos en el apartado anterior.

\subsection{Ensayos de inmersión total en laboratorio}

Los ensayos de inmersión en laboratorio se han realizado de acuerdo con las recomendaciones de la norma ASTM G-31.

Las probetas, antes de cada ensayo, se limpiaron con agua destilada y se secaron con alcohol y acetona. Se mantuvo una relación volumen de disolución/superficie del electrodo de $20 \mathrm{~mL} / \mathrm{cm}^{2}$ y la temperatura, termostatizada a $23 \pm 1{ }^{\circ} \mathrm{C}$.
Los productos de corrosión se eliminaron por inmersión en una solución de $\mathrm{NH}_{4} \mathrm{Cl}(100 \mathrm{~g} / \mathrm{L})$ a $70{ }^{\circ} \mathrm{C}$ durante un período de 2 a $3 \mathrm{~min}$.

\subsection{Ensayos electroquímicos}

Los ensayos electroquímicos se llevaron a cabo en una celda de 3 electrodos, utilizándose, como referencia, uno de calomelanos saturado (ECS) y como contraelectrodo, uno de grafito. La superficie de trabajo fue de $1 \mathrm{~cm}^{2}$. Los electrodos se conectaron a una tarjeta potenciostática modelo GamryCMS 105, con software adecuado para la adquisición y procesamiento de los datos. Las curvas potenciodinámicas se realizaron partiendo del potencial catódico de $-50 \mathrm{mV}$ (referido al potencial de corrosión) y barriendo en la dirección anódica a una velocidad de $0,16 \mathrm{mV} / \mathrm{s}$, hasta alcanzar una densidad de corriente de $1 \mathrm{~mA} / \mathrm{cm}^{2}$.

\subsection{Análisis de superficie}

Para el examen SEM, se utilizó un equipo Jeol JXA 840 con dos canales de longitud de onda acoplados y un sistema de análisis de dispersión de energía (EDS).

\section{RESULTADOS EXPERIMENTALES Y DISCUSIÓN}

\subsection{Adición del inhibidor al medio}

En la figura 1 se presentan los diagramas de polarización obtenidos para el acero galvanizado en ausencia y presencia de concentraciones variables de inhibidor (0-1.000 ppm). La adición de cerio al medio ha modificado sensiblemente el comportamiento electroquímico del acero galvanizado. Se aprecia un desplazamiento del potencial de corrosión de unos $30 \mathrm{mV}$ hacia valores más activos, una clara reducción en los valores de densidad de corriente catódica y la aparición de un punto de inflexión en la rama anódica. Este comportamiento difiere sensiblemente del que presenta el acero galvanizado, sin adición de cerio, donde la rama anódica presenta una activa disolución del cinc y la catódica un control límite de difusión de oxígeno.

En la tabla I se presentan los principales parámetros electroquímicos y grado de protección producido por el inhibidor, calculados a partir del análisis de las curvas en porcentaje respecto a la velocidad de corrosión del acero galvanizado en la disolución sin adición de inhibidor. Estos datos son coherentes con los presentados en la tabla II, que 


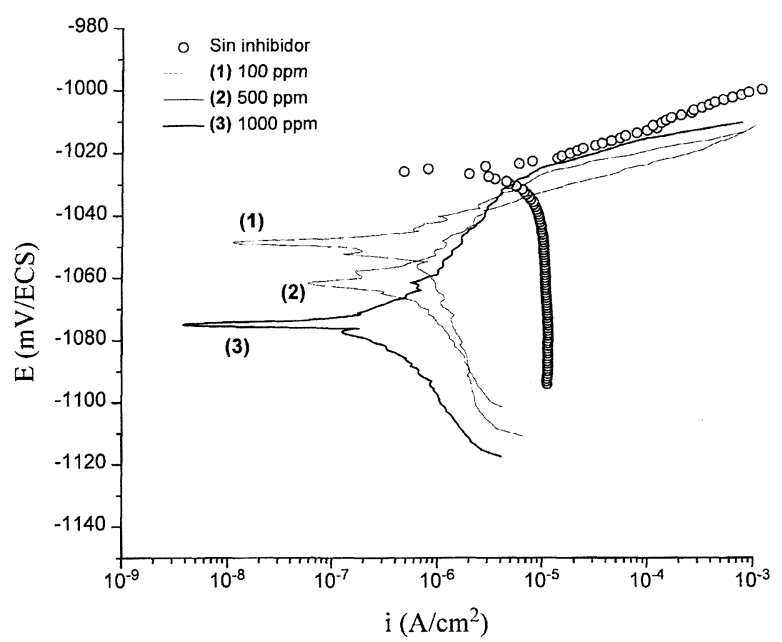

FIG. 1.- Diagramas de polarización lineal en disoluciones aireadas en $\mathrm{NaCl}$ 3,5\% con concentraciones crecientes de $\mathrm{CeCl}_{3}$.

FIG. 1.- Linear polarisation diagrams in $\mathrm{NaCl}$ $3.5 \%$ aerated solutions with different $\mathrm{CeCl}_{3}$ concentrations.

recogen los valores medios de pérdida de masa (g/día) y de velocidad de corrosión (mm/año) obtenidos en los ensayos de inmersión completa, en períodos de tiempo comprendidos entre 48 y $192 \mathrm{~h}$, así como con los datos bibliográficos recogidos en (8), donde se da como rango de velocidades típicas de corrosión de aceros galvanizados en diferentes tipos de agua de mar 0,12-0,25 mm/año.

\subsection{Pretratamientos}

En la bibliografía, es frecuente encontrar estudios tendentes a determinar cómo el cerio se incorpora a la superficie del material pretratado, con objeto de poder definir su mecanismo de actuación. Así, en (9) se identifican los compuestos intermetálicos ricos en cobre situados sobre la superficie como centros catódicos, donde, de forma aislada, se desarrolla la película de cerio. De forma parecida, en (10) son los precipitados $\mathrm{Mn}-\mathrm{Fe}$ los puntos más catódicos de la aleación AA5083.

En el presente trabajo, las probetas de acero galvanizado pretratadas en sales de cerio, presentaron a simple vista un recubrimiento fino y continuo de coloración amarilla. En la micrografía SEM de la figura 2, se ve, en su superficie, una distribución al azar de depósitos circulares de $50 \mu \mathrm{m}$ de diámetro. A mayores aumentos (Fig. 3), se distingue que éstos están constituidos por la acumulación de pequeñas partículas esféricas distribuidas en forma radial y adheridas sobre la superficie, la cual se presentó cuarteada después del proceso de secado. Los perfiles EDS de la figura $4 a$ y $b$ demuestran que existe incorporación de cerio, tanto en las zonas externas como en las partículas que constituyen los depósitos (zonas 1 y 2 de la figura 3), pero con unos niveles de concentración muy distintos, según indican las alturas de los picos característicos del cerio. Este hecho confirma la existencia de un mecanismo de deposición catódico, de tal forma que sobre determinados puntos de la superficie se desarrollan los centros de reducción de oxígeno, aumentando localmente la alcalinidad y precipitando posteriormente el cerio en forma de óxido y/o hidróxido.

Para evaluar la mayor resistencia a la corrosión de los recubrimientos obtenidos se empleó el ensayo acelerado de inmersión alternada. La agresividad de este ensayo es bastante mayor que la que

TABla II.- Pérdida de peso, velocidad de corrosión y grado de protección, determinados a partir de los ensayos de inmersión completa

TABLE II.-Corrosion rate (measured by weight loss) and protection rate, obtained from complete inmersion experiments

\begin{tabular}{|c|c|c|c|}
\hline Medio & $\Delta \mathrm{W}$ (g/día) & Corr (mm/año) & $\mathrm{P} \%$ \\
\hline $\mathrm{NaCl} 3,5 \%$ & 0,0390 & 0,2139 & 0 \\
\hline $\mathrm{NaCl} 3,5 \%+1000 \mathrm{ppm}$ & 0,0014 & 0,0074 & 96,5 \\
\hline
\end{tabular}

TABLA I.- Parámetros electroquímicos y grado de protección aportado para distintas concentraciones de $\mathrm{CeCl}_{3}$. Valores obtenidos a partir de los diagramas de polarización

TABLE I.-Electrochemical parameters and protection degree afforded by different $\mathrm{CeCl}_{3}$ concentrations. Data obtained from polarisation diagrams

\begin{tabular}{|c|c|c|c|c|}
\hline Medio & Ecorr $(\mathrm{V})$ & Icorr $\left(\mathrm{A} / \mathrm{cm}^{2}\right)$ & Corr $(\mathrm{mm} /$ año $)$ & $\mathrm{P} \%$ \\
\hline $\mathrm{NaCl} 3,5 \%$ & $-1,031$ & $7,05 \mathrm{E}-06$ & 0,106 & 0 \\
$\mathrm{NaCl} 3,5 \%+100 \mathrm{ppm}$ & $-1,056$ & $6,08 \mathrm{E}-07$ & 0,009 & 91,4 \\
$\mathrm{NaCl} 3,5 \%+200 \mathrm{ppm}$ & $-1,052$ & $4,41 \mathrm{E}-07$ & 0,007 & 93,8 \\
$\mathrm{NaCl} 3,5 \%+500 \mathrm{ppm}$ & $-1,062$ & $2,84 \mathrm{E}-07$ & 0,004 & 96,0 \\
$\mathrm{NaCl} 3,5 \%+1000 \mathrm{ppm}$ & $-1,066$ & $3,60 \mathrm{E}-07$ & 0,005 & 94,9 \\
\hline
\end{tabular}




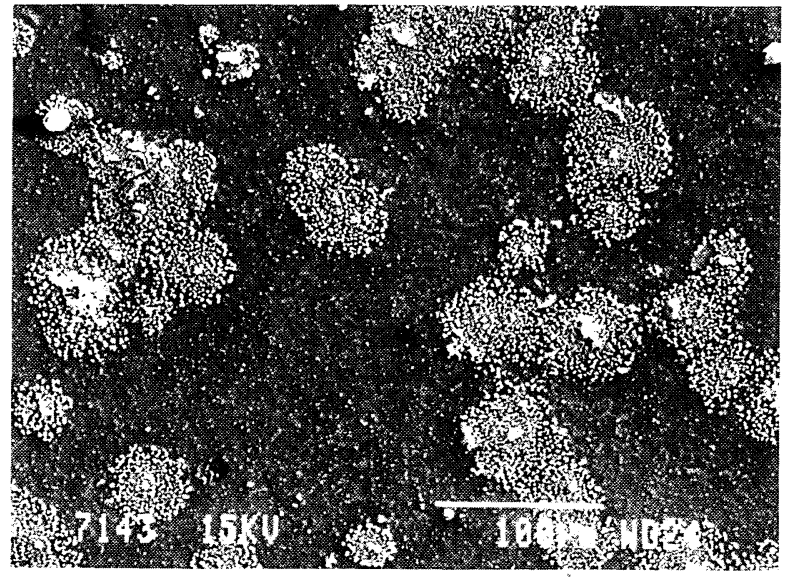

FIG. 2.- Micrografía SEM de la superficie después de pretratamiento en $\mathrm{NaCl} 3,5 \%-1000$ ppm $\mathrm{CeCl}_{3}$.

FIG. 2.-SEM micrograph of surface after pretreatings in $\mathrm{NaCl} 3.5 \%-1000 \mathrm{ppm} \mathrm{CeCl}_{3}$.

presentan los ensayos de inmersión completa debido a la presencia continuada de una capa de electrólito altamente aireada. Se tomó, como criterio de evaluación, el tiempo necesario hasta la aparición del "primer punto blanco" (producto de corrosión característico del zinc). Mientras que las probetas que carecían de tratamiento presentaron "punto blanco" a las $3 \mathrm{~h}$ del inicio, las pretratadas necesitaron 96 h para su aparición. En las figuras 5 y 6 se aprecia el estado superficial de ambas probetas después de las $96 \mathrm{~h}$ de ensayo.

En la figura 7 se comparan los diagramas de polarización de las probetas pretratadas junto con las

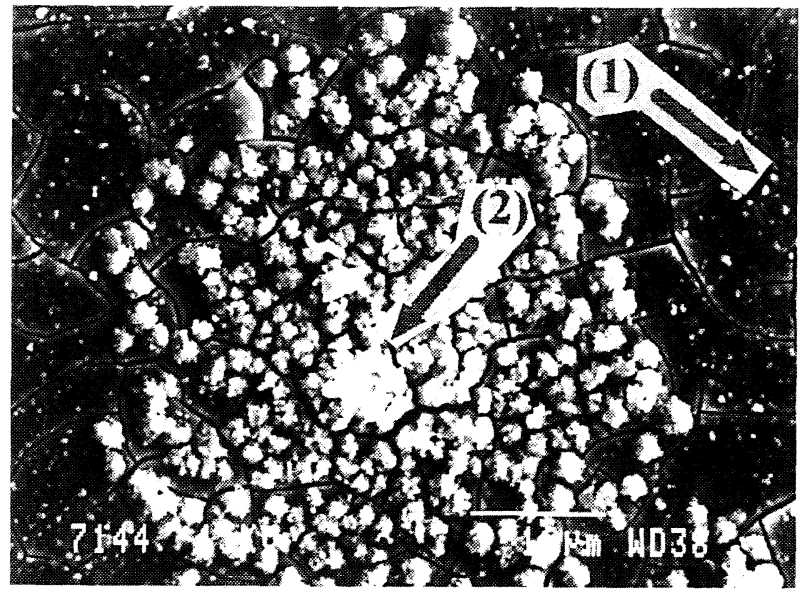

FIG. 3.- Detalle a alta magnificación de uno de los depósitos formados sobre la superficie.

FIG. 3.- High magnification detail of one of the deposits formed on surface.

curvas obtenidas al añadir $1.000 \mathrm{ppm}$ de inhibidor al medio y de acero galvanizado sin inhibir. Las probetas pretratadas muestran una clara reducción en la velocidad de corrosión, aportando una protección del $87 \%$, pero sin llegar a los niveles obtenidos en la tabla I.

\section{CONCLUSIONES}

- El tricloruro de cerio, añadido al medio o mediante pretratamiento proporciona un alto grado de protección contra la corrosión de aceros

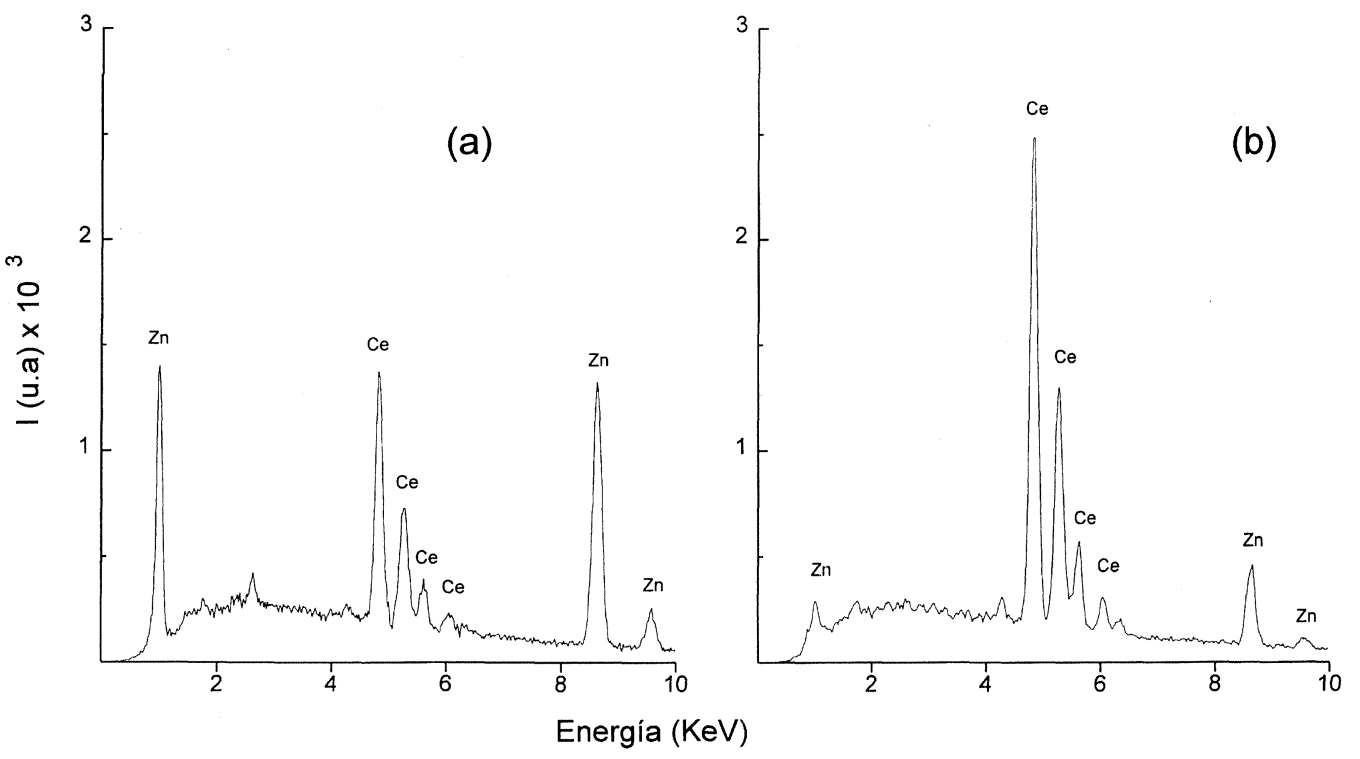

FIG. 4.- Espectros EDS adquiridos (a) sobre zona 1 y (b) sobre zona 2 de la micrografía SEM de la figura 3. FIG. 4.- EDS spectra (a) on zone 1 and (b) on zone 2 of SEM micrograph in figure 3. 


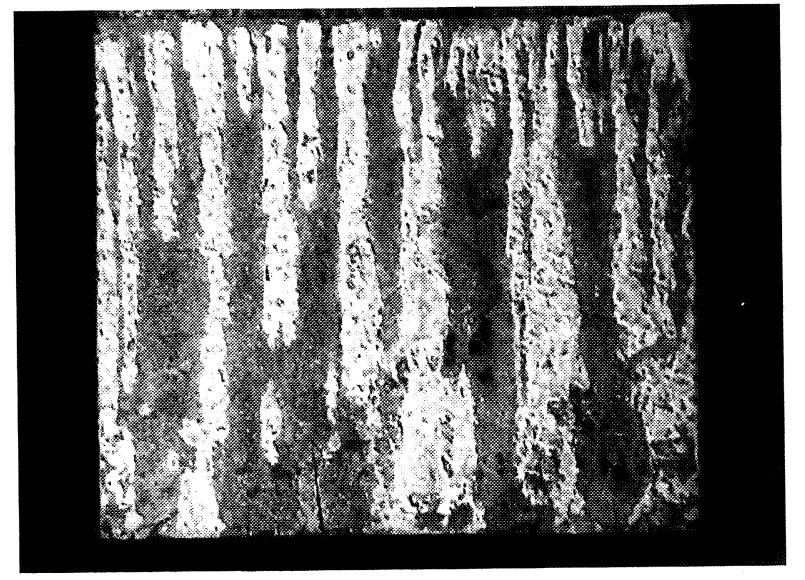

FIG. 5.- Fotografía de la superficie del acero galvanizado, sin pretratamiento, después de $96 \mathrm{~h}$ de ensayo de inmersión alternada en $\mathrm{NaCl}$.

FIG. 5.- Photograph of the galvanised steel surface, without pre-treatment, after $96 \mathrm{~h}$ in alternate inmersion test.

galvanizados en medios de $\mathrm{NaCl}$ al 3,5\% saturado en oxígeno.

- La formación de una película rica en cerio, desarrollada sobre los centros catódicos, es la responsable de estas mejoras.

- El tiempo de pretratamiento utilizado $(60 \mathrm{~h})$, debe reducirse hasta alcanzar valores de interés para la industria. En este sentido, se sigue trabajando con el objeto de conseguir la aplicación de pretratamientos anticorrosivos no contaminantes que exijan bajos tiempos de aplicación.

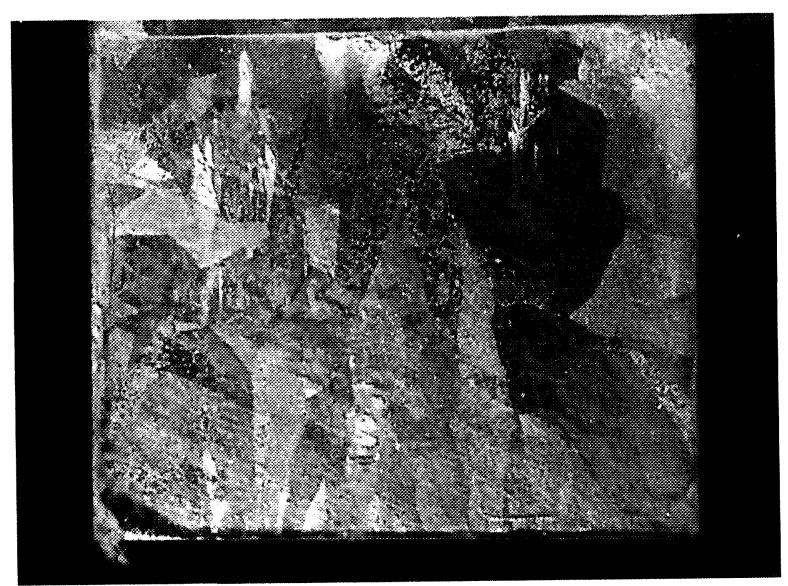

Fig. 6.- Fotografía de la superficie del acero galvanizado, pretratado en $\mathrm{CeCl}_{3}$, después de $96 \mathrm{~h}$ de ensayo de inmersión alternada en $\mathrm{NaCl}$.

FIG. 6.- Photograph of the galvanised steel surface, pre-treated in $\mathrm{CeCl}_{3}$, after $96 \mathrm{~h}$ in alternate inmersion test.

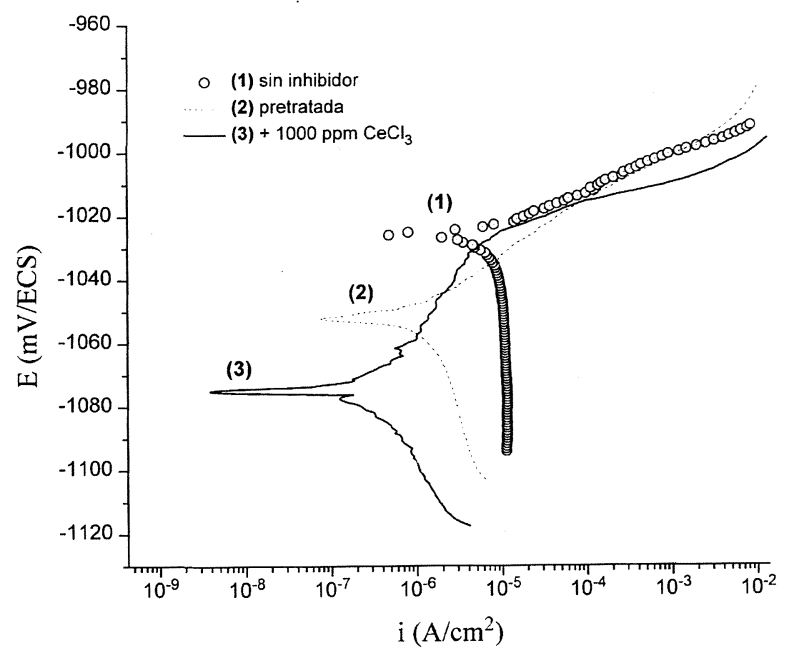

FIG. 7.- Diagramas de polarización lineal de acero galvanizado en disoluciones aireadas de $\mathrm{NaCl}$. (1)

sin inhibidor. (2) pretratada. (3) $+1000 \mathrm{ppm}$.

FIG. 7.- Linear polarization diagrams of galvanised steel in $\mathrm{NaCl}$ aerated solutions. (1) without inhibitor. (2) pre-treated. (3) +1000 ppm.

\section{Agradecimiento}

Los autores desean agradecer a la CICYT la financiación del proyecto "Tratamientos superficiales de bajo impacto medio ambiental. Sistemas basados en elementos lantánidos" (MAT97-1075-003-02) dentro del cual se ha realizado el presente trabajo.

\section{REFERENCIAS}

(1) PORTER, F.C. Corrosion resistance of zinc and zinc alloys. $1^{\text {a }}$ ed. (Eds. A. Philip y P.E. Schwietzer. Nueva York (EE.UU.) 1994: 39.

(2) Arnott, D.R., Hinton, B.R.W. y Ryan, N.E. Corrosion. 45, 1989: 12-18.

(3) Mansfeld, F. y Wang, Y. Br. Corros. J. 29 (3), 1994: 194-200.

(4) Marcos, M. Pretratamientos anticorrosivos no contaminantes. Nuevos métodos basados en sales lantánidas. Tesis doctoral. Universidad de Cádiz. 1997.

(5) Lu, Y.C. e Ives, M.B. Corros. Sci., 37 (1), 1994: 145155.

(6) Breslin, C.B., Chen, C. y Mansfeld, F. Corros. Sci., 39 (6), 1997: 1.061-1.073.

(7) Hinton, B.R.W. y Wilson, L. Corros. Sci., 29 (8), 1989: 967-985.

(8) Ver 1). Pag. 316.

(9) Aldykewicz, A.J., Isaacs, H.S. y Davenport, A.J. $J$. Electrochem. Soc. 142 (10), 1995: 3.342-3.350.

(10) Botana, F.J., Aballe, A., Bethencourt, M., Marcos, M., Pérez, J. y Rodriguez, M.A. Proc. Eurocorr 97. Trondheim (Noruega). 1997: 339-344. 